\title{
Development of a sensor-based approach for local minima recovery in unknown environments
}

\begin{abstract}
This paper introduces a new methodology for escaping from local minima using an actualvirtual target switching strategy. In particular, this approach proposes suitable steps to detect trap situations and guide the robot away from local minima even when the environment is completely unknown. In this work the navigation system consists of two layers. In the lowlevel layer, a Nearest Virtual Target (NVT) approach is adapted as a reactive collision avoidance method for mobile robot navigation to achieve collision free motion in cluttered, dense and troublesome scenarios. Where the robot is surrounded by obstacles and a trap situation is likely to occur, the high-level layer becomes responsible to plan a path to pull the robot out of the trap. Finally, the performance of the proposed approach is validated by simulation results.
\end{abstract}

Keyword: Mobile robots; Sensor-based navigation; Obstacle avoidance; Virtual target; Fuzzy control; Path planning 